\title{
Editorial
}

\section{Acknowledgement to Reviewers of Marine Drugs in 2013}

Marine Drugs Editorial Office, MDPI AG, Klybeckstrasse 64, CH-4057 Basel, Switzerland

Received: 20 February 2014 / Published: 24 February 2014

The editors of Marine Drugs would like to express their sincere gratitude to the following reviewers for assessing manuscripts in 2013:

\begin{tabular}{|c|c|c|}
\hline Abdelmohsen, Usama Ramadan & Arias, Hugo & Bates, Stephen \\
\hline Abreu, Pedro & Arias, Maria Cecilia & Battaglia, Luigi \\
\hline Adachi, Masao & Arkhipova, Irina R. & Baughn, Anthony D. \\
\hline Adams, Nick & Asai, Teigo & Becker, Pierre \\
\hline Afiyatullov, Shamil Sh. & Asakawa, Yoshinori & Bedini, Emiliano \\
\hline Agbaga, Martin-Paul & Aytekin, A. Ozhan & Bellei, Barbara \\
\hline Agyei, Dominic & Ayyalusamy, Ramamoorthy & Beniash, E. \\
\hline Aitken, Robert John & Azadi, Parastoo & Benkendorff, Kirsten \\
\hline Akk, Gustav & Azam, Layla & Ben-Shabat, Shimon \\
\hline Alfonso, Amparo & Azuma, Kazuo & Berdyshev, Evgeny \\
\hline Alloatti, Giuseppe & Bagno, Alessandro & Beretta, Giovanni \\
\hline Alonso, Alejandra & Baker, Bill & Berger, Walter \\
\hline Alvarez, Ana I. & Baldwin, Susan A. & Bergès, Thierry \\
\hline Alves, Anabela & Balskus, Emily P. & Bero, Joanne \\
\hline Amade, Philippe & Baltz, Richard H. & Berry, John P. \\
\hline Amy, Penny & Balunas, Marcy J. & Berta, Giovanni Nicolao \\
\hline Anderson, Clarissa R. & Bandarra, Narcisa Maria & Biagini, Giuseppe \\
\hline Andjelkovic, Anuska V. & Banerjee, Debabrata & Bickmeyer, Ulf \\
\hline Andolfi, Anna & Banerjee, Monimoy & Bilan, Maria \\
\hline Andrade, Paula Branquinho & Bankova, Vassya & Bisignano, Giuseppe \\
\hline Andrianasolo, Eric & Banoub, Joseph H. & Blanchfield, Joanne \\
\hline Angeles, Thelma S. & Banskota, Arjun H. & Blázquez, María \\
\hline Anraku, Makoto & Baptista, Mafalda S. & Blunt, John W. \\
\hline Apáti, Ágota & Barbosa, Teresa M. & Bøgwald, Jarl \\
\hline Arai, Masayoshi & Bargu, Sibel & Bohlin, Lars \\
\hline Arbogast, Sandrine & Basti, Leila & Bondon, Arnaud \\
\hline
\end{tabular}


Bongarzone, Salvatore

Bonheyo, George

Bontemps, Nataly

Borchman, Douglas

Bosmans, Frank

Botana, Luis

Bourdelais, Andrea

Bourdon, J.A.

Bowden, Bruce

Boyle, Joseph J.

Braig, Simone

Brase, Stefan

Brash, A.R.

Breitling, Rainer

Brett, Michael T.

Bright, J.J.

Brinkman, Diane L.

Broggini, Massimo

Bruhn, Torsten

Brunet, Christophe

Bruno, Laura

Bruno, M.

Büttner, Sabrina

Bugni, Tim S.

Buisson, Didier

Burgos, Javier S.

Butler, Mark

Butler, Mark S.

Butts, Craig P.

Bykov, Vladimir J.N.

Cabado, Ana G.

Cabot, Myles

Cabrita, Maria Teresa

Calado, Ricardo

Calder, Philip

Caldwell, Gary

Caldwell, Kim A.

Cammarata, Matteo

Canki, Mario

Capper, Angela

Cardoso, Susana M.

Carmeli, Shmuel
Carrano, Lucia

Carter, Darrick

Casapullo, Agostino

Casely-Hayford, Maxwell

Castell, Alina

Castro, Ana

Cavalieri, Vincenzo

Cavalli, Roberta

Cavanagh, John

Celik-Ozenci, Ciler

Ceron, Jose

Chabert, Philippe

Chai, Sergio C.

Chakrabarti, Debopam

Chang, Fang-Rong

Chang, Wen-Cheng

Châtel, Amélie

Chen, Bing

Chen, Jih-Jung

Chen, Jin-Jer

Chen, Qian

Chen, Qi-Yin

Chen, Shawn

Cheng, Shi-Yie

Cheng, Shu

Cheng, Yuan-Bin

Cherian, Philip

Chew, Boon

Choi, Hong Seok

Choi, Jong-il

Choi, Yung Hyun

Chooi, Yit-Heng

Christaki, Efterpi

Christie-Oleza, Joseph

Chung, Chuhan

Chung, Hun-Taeg

Church, W. Bret

Cianca, Rosa C. Cervantes

Ciapetti, Gabriela

Ciardelli, Gianluca

Ciavatta, Maria Letizia

Ciufolini, Marco
Clark, Richard

Clericuzio, Marco

Codd, Rachel

Collado, Isidro G.

Concannon, Caoimhin

Condezo-Hoyos, L.

Cooksey, Keith

Cooper, Edwin L.

Cooper, William T.

Cordoba-Diaz, D.

Corsaro, Maria Michela

Corte-Real, Manuela

Costa-Lotufo, Letícia V.

Costantini, Maria

Costantino, Valeria

Costa-Rodrigues, João

Coyne, Kathryn

Cresteil, Thierry

Cretoiu, Mariana Silvia

Cristina, Teixidó

Cudic, Mare

Curnow, Paul

Cusick, Kathleen D.

D'abrosca, Brigida

D'ursi, Pasqualina

D'Acquisto, Fulvio

Dale, Brian

Daly, Norelle

Damodaran, Vinod Babu

Dangardt, Frida

Dattelbaum, Jonathan D.

Davenport, Ian R.

De Agostini, Ariane

De Castro, Cristina

De Felício, Rafael

De Koning, Harry P.

De La Vega, Ricardo R.

De Lera, Angel R.

De Marino, Simona

De Mendonça, Dina I.M.D.

De Morais, Rui Manuel Santos

Costa 
De Pascale, Donatella

De Riccardis, Francesco

De Rosa, Salvatore

De Troch, Marleen

Deeds, Jonathan R.

Delfourne, Evelyne

Demadis, Kostas

Desaubry, Laurent

Desideri, Alessandro

Devenish, Rod

Diana, Patrizia

Díaz-Marrero, Ana R.

Dickschat, Jeroen S.

Dimas, Konstantinos

Diogene, Jorge

Dittmann, Elke

Doerrler, William

Domínguez, Elena

Domínguez, Herminia

Dong, Jixin

Dooley, Helen

Dornetshuber, Rita

Doroghazi, James R.

Døskeland, Stein

Downing, Tim

Dreanno, Catherine

Drewry, David H.

Duarte, Noélia

Duh, Chang-Yih

Durkin, Colleen

Durnford, Dion

Dwivedi, Chandradhar

Eberhart, Bich-Thuy L.

Edison, Arthur S.

Edrada-Ebel, RuAngelie

Edwards, Andrea

Egan, Suhelen

Eirín-López, José M.

Elsebai, Mahmoud Fahmi

Elsinghorst, Paul W.

Ely, B.

Emblem, Åse
Enomoto, Keiich

Eriksson, Mats

Espiña, Begoña

Esteves, Ana I.S.

Evans, Bradley S.

Ewart, H. Stephen

Fahmy, Hesham T.Y.

Falconer, Ian R.

Falkowski, Paul

Falson, Pierre

Faraoni, David

Farrell, Hazel

Fassouane, Aziz

Faulkner, Simon

Fecik, Robert

Fedorenko, Victor

Feng, Rentian

Férézou, Jean-Pierre

Fernández, José J.

Fewer, David

Fiebig, Heinz Herbert

Figueroa, Félix López

Fisher, Aron B.

Fitton, Janet Helen

Fleming, Lora

Florence, Gordon J.

Florent, Isabelle

Florio, Tullio

Flower, Darren

Forano, Evelyne

Forim, Moacir Rossi

Forzato, Cristina

Foster, Michelle T.

Frame, Elizabeth

Franco, Chris

Franco, José M.

Franco, Octavio

Frank, Uri

Franzetti, Andrea

Freeman, Christopher J.

Freitas, Ana C.

Frisvad, Jens Christian
Frohbergh, Michael

Froscio, Ssuzanne M.

Fuentes-Grünewald, C.

Fuwa, Haruhiko

Gaboury, Louis

Gademann, Karl

Galano, Jean-Marie

Gallo, Alessandra

Galluzzi, L.

Gandour, Richard

Ganesan, A.

Garcia-Cuetos, Lydia

Garner, Amanda

Gazdar, Adi

Gbogouri, Albarin

Gelman, Faina

Genilloud, Olga

Genovese, Giuseppa

George, Sarah J.

Getchell, Rodman

Ghayur, Muhammad Nabeel

Gilmour, Jim

Glaser, Keith B.

Glencross, Brett

Gochfeld, Deborah

Goh, Beverly Pi Lee

Gomez-Escribano, Juan-Pablo

Gordaliza, M.

Gottesman, Michael M.

Grabarczyk, Małgorzata

Gram, Lone

Grassi, Giovanni

Gray, Christopher A.

Green, David W.

Gregory, Melissa K.

Grenha, Ana

Grkovic, Tanja

Grogan, Gideon

Grovel, Olivier

Gugger, Muriel

Gugliandolo, Concetta

Gulder, Tobias 
Gupta, Prasoon

Gupta, Subash C.

Guschina, Irina A.

Gustafson, Kirk

Guzmán, Esther A.

$\mathrm{Ha}, \mathrm{Ki}-\mathrm{Tae}$

Häder, Donat-P.

Haft, Daniel H.

Hagenbuch, Bruno

Hajduch, Eric

Hallegraeff, Gustaaf M.

Hallsworth, J.E.

Hanif, Novriyandi

Hansen, Espen

Hanski, Leena

Harder, Tilmann

Harinantenaina, Liva

Harnedy, Pádraigín

Hashidoko, Yasuyuki

Hawinkels, Lukas J.A.C.

Hayashi, Masahiro

Hayen, Heiko

Helbert, William

Hellmich, Christian

Helm, Richard F.

Helms, Volkhard

Henrich, Curtis J.

Henriques, Sonia

Heo, Soo-Jin

Heretsch, Philipp

Herfindal, Lars

Hernández-Losa, Javier

Herr, Ingrid

Herraiz, Tomás

Herrero, M.

Hidari, Kazuya I.P.J.

Hildebrand, Mark

Hintze, Vera

Hirokawa, Takatsugu

Hoeger, Ulrich

Hohensinner, Philipp

Holland, Patrick
Holmes, Philip

Holst, Otto

Honoré Hansen, Steen

Hopkins, Ann

Hoshi, Naoto

Hosokawa, Masashi

Hou, Guangjin

Hou, Yanpeng

Howie, Sarah

Hu, Youcai

Huang, Dorothy $\mathrm{Yu}$

Huang, Shengxiong

Huang, Shi-Ying

Huet, Eric

Hughes, Francis M.

Huigens III, Robert W.

Hungerford, James

Hunter, Christy

Hwang, Tsong-Long

Ibanez, Elena

Imhoff, Johannes

Ioannou, Efstathia

Irhimeh, Mohammad R.

Ishibashi, Masami

Ishiki, Manabu

Iskandarov, Umidjon

Ito, Takeo

Ito, Tatsuya

Itoi, S.

Iwagawa, Tetsuo

Iwao, Masatomo

Jachymek, Wojciech

Jacobson, Peer

Jansen, Rolf

Jeannerat, Damien

Jégou, Camille

Jeon, You-Jin

Jesus Raposo, Maria Filomena

Jiang, Lin-Hua

Jiang, Sunny

Jiao, Guangling

Jiménez-Barbero, Jesús
Jiménez, Carlos

Jin, Zhendong

Joo, Hong-Gu

Jos, A.

Kaasalainen, Ulla

Kalaitzis, John

Kalimuthu, Senthilkumar

Kalinin, Vladimir

Kan, Toshiyuki

Kaneko, Jun

Kashman, Yoel

Kaufmann, Katrin

Kawai, Shigeyuki

Kawakami, Koji

Kawasaki, Tomomi

Kearns, Philip S.

Keatinge-Clay, Adrian

Kellenberger, Esther

Kellenberger, Stephan

Kelley, Darshan S.

Kellmann, Ralf

Kelly, Wendy

Kem, William

Kenttämaa, Hilkka I.

Kerr, Steve

Khadem, Shahriar

Khanna, Rajesh

Khozin-Goldberg, Inna

Kiene, Ronald P.

Kikuchi, Haruhisa

Kilcoyne, Jane

Kim, Gwang Hoon

Kim, Hye Kyong

Kim, Ji Hoe

Kim, Paul

Kim, Se-Kwon

Kim, Sung-Hoon

Kinnear, Susan

Kinsey, William H.

Kishimoto, Yoshimi

Kita, Yasuyuki

Kletsas, Dimitris 
Klint, Julie

Kluza, Jerome

Knölker, Hans-Joachim

Kobayashi, Makito

Kobayashi, Yasuhisa

Köcher, Thomas

Kodani, Shinya

Kolaczkowski, Marcin

Komoroski, Richard A.

Kong, Fangming

Konoki, Keiichi

Kornprobst, Jean Michel

Korsnes, Mónica Suárez

Koshino, Hiroyuki

Koskinen, Ari M.P.

Kotoku, Naoyuki

Kourist, Robert

Kovbasnjuk, Olga

Kraan, Stefan

Krepkiy, Dmitriy V.

Krock, Bernd

Krüger, Thomas

Kubo, Yoshinao

Kubota, Takaaki

Kuczer, Mariola

Kujawinski, Elizabeth B.

Kumirska, Jolanta

Kuo, Jen-Min

Kuroda, Chiaki

Kurosawa, Norio

Kurtboke, Ipek

Kwon, Young M.

Lai, Daowan

Laird, Damian

Lamarche, Matthew J.

Lampert, Angelika

Landini, Paolo

Lane, Amy L.

Lang, Siegmund

Lanzetta, Rosa

Latortue, Marie C.

Lau-Cam, Cesar A.
Lauder, Bob

Laurienzo, Paola

Lauritano, Chiara

Lay, Horng-Liang

Laycock, Maurice V.

Leal, Miguel

Lebeau, Thierry

Leblond, Jeffrey

Lee, Sang Kook

Lee, Seung-Min

Lee, Wen-Sen

Lefay, Catherine

Lehotay, Steven J.

Lehrer, Robert I.

Leikoski, Niina

Leone, Antonella

Lesueur, Celine

Lewandowski, $\mathrm{Pa}$

Li, Huabin

$\mathrm{Li}, \mathrm{Ke}$

Li, Lu-Ping

Liermann, Johannes C.

Lim, Yun-Ping

Lin, Zhicheng

Linder, Stefan

Lindholm, Dan

Liras, Paloma

Litaker, Wayne

Littlefield, Bruce A.

Liu, Delong

Liu, Jin

Llewellyn, Carole

Long, Benedict $\mathrm{M}$.

Longstaff, Colin

Lönnberg, Harri

Lopanik, Nicole B.

Lopata, Andreas L.

Lopez-Huertas, Eduardo

Lordan, Sinéad

Lu, Chung-Kuang

Lu, Jun

Lundholm, Nina
Lurling, Miquel

Lutz, Richard

Ma, Edmond Dik-Lung

MacMillan, John B.

Madeo, Frank

Madhukar, Burra

Maeda, Hayato

Maes, Louis

Maier, Jeanette A.M.

Maisch, Tim

Majik, Mahesh

Malgesini, Beatrice

Maloney, Katherine

Mamoun, Choukri Ben

Mancini, Ines

Mándi, Attila

Manfrin, Chiara

Mangoni, Alfonso

Månsson, Maria

Manzo, Emiliano

Mao, Simon J.T.

Marchetti, Philippe

Marchioli, Roberto

Marco-Contelles, José

Mariottini, Gian Luigi

Markopoulos, John

Marron, Alan O.

Martinez, Ronny

Martínez-Banaclocha, Marcos

Arturo

Martinez, Ana

Másson, Már

Mastinu, Andrea

Mata, Leonardo

Matsuda, Fumio

Matsunaga, Shigeki

Matsuya, Yuji

Matthews, Jacqueline M.

Mazurak, Vera

Mazzei, Pierluigi

McAlpine, Shelli

Mcarthur, Jeff 
Mccarthy, Peter

McCarty, Mark

Mcdougal, Owen M.

Mcgregor, Glenn B.

Mckee, Lauren S.

Mclean, Timothy I.

Mcmanus, Owen B.

Mebs, Dietrich

Medina, Miguel A'ngel

Mehiri, Mohamed

Menna, Marialuisa

Mi, Fwu-Long

Mitchell, John

Miyaoka, Hiroaki

Mizuno, Masashi

Moczydlowski, Edward G.

Moffit, Michelle

Molgo, Jordi

Molinski, Tadeusz

Mollica, Adriano

Molnár, István

Montero, Olimpio

Montresor, Marina

Monzavi-Karbassi, Behjatolah

Moody, A. John

Moody, Christopher J.

Moody, Terry W.

Moore-Kucera, Jennifer

Morabito, Marina

Moran, Yehu

Moreira, Susana

Moreno, Andrés

Mori, Kenji

Mori, Naoki

Morikawa, Toshio

Morimura, Shigeru

Morton, Steve L.

Morzycki, Jacek W.

Moscow, Jeffrey A.

Motti, Cherie

Moussa, Fathi

Müller, Werner E.G.
Munday, Rex

Muramoto, Koji

Murphy, Karen

Murphy, Patrick J.

Muylaert, Koenraad

Nacke, Heiko

Nagai, Hiroshi

Nair, Satish K.

Naka, Hiroaki

Nakao, Yoichi

Napier, Johnathan

Nappo, Michela

Navacchia, Maria Luisa

Nestor, Carballeira

Neuzil, Jiri

Newman, David

Nguyen, Anh-Tho

Nicoletti, Rosario

Nielsen, Thorbjørn Terndrup

Nijland, Reindert

Niki, Hironori

Nissen-Meyer, Jon

Nobili, Stefania

Noguchi, Tamao

Nordon, Robert E.

Norte, Manuel

Norte, Munuel

Northen, Trent

Novelli, Antonello

Numata, Keiji

Oberlies, Nicholas H.

Oberthür, Markus

Ochi, Kozo

Oda, Tatsuya

O'doherty, George

Oh, Dong-Chan

Ohta, Shinji

Oishi, Shinya

Ojida, Akio

Ojika, Makoto

Ok, Salim

Okada, Seiji
Okamoto, Susumu

Okamoto, Yoshiharu

Okello, Edward

Olafsdottir, Elin Soffia

Oliva, M.

Oliveira Rocha, Hugo Alexandre

Olsen, Christian

Omarsdottir, Sesselja

Ontsouka, Edgar Corneille

Opatz, Till

Ortíz, Abel Baerga

Osaki, Tomohiro

Ostenfeld Larsen, Thomas

Östman, Örjan

Ouahid, Youness

Ovenden, Simon

Ovissipour, Mahmoudreza

Pagliara, Patrizia

Pahwa, Savita

Pallela, Ramjee

Palomares, Eva Rufino

Palumbo, Anna

Park, Joo-In

Park, Wansu

Parrington, John

Passarelli, Marisa

Pawel, Pawel

Payo, Dioli Ann

Pearce, A. Norrie

Pei, Jin-Jing

Pelkonen, Olavi

Pelletier, Jerry

Pemberton, John M.

Peng, Hanjing

Peng, Jiang-Nan

Penna, Antonella

Pereira Neto, Ana Maria

Pereira, Leonel

Pérez, Andy J.

Pérez-Victoria, Ignacio

Perhar, Gurbir

Perrone, Daniela 
Pescitelli, Gennaro

Pestov, A.V.

Petrie, James

Pfeifer, Gerd

Pflugmacher, Stephan

Picot, Laurent

Piel, Jörn

Pietro, A. Di

Piggott, Andrew

Piorko, Adam

Pires, O.R.

Pistocchi, Rossella

Pitsinos, Emmanuel N.

Plouguerné, Erwan

Podechard, Normand

Podlasek, Carol A.

Pohnert, Georg

Pomati, Francesco

Pomin, Vitor Hugo

Pottinger, Tom G.

Pouchus, Yves François

Prêcheur, Isabelle

Prescott, Mark

Proksch, Peter

Proteau, Philip J.

Quesada, Ana R.

Quesada, António

Quilliam, Michael A.

Quinton, Loïc

Radwan, Mohamed M.

Raine, Robin

Ramis, Joana

Ramón Y. Cajal, Santiago

Rasapalli, Sirappa

Rateb, Mostafa E.

Rawson, Ashish

Reft, Abigail

Rein, Kathleen

Reitzel, Adam

Remias, Daniel

Reyes, Fernando

Rho, Jung-Rae
Rialland, Mickaël

Ribalet, Francois

Riccio, Raffaele

Rice, Scott

Rienzi, Sara Di

Robertson, Charles R.

Robinson, Mark

Rocchetti, Marcella

Rocha-Santos, Teresa A.P.

Rodrigues, Alice

Rodriguez, Carmen

Rodríguez, Ileana I.

Rodriguez, Jaime

Rohan, Lisa Cencia

Rohrlack, Thomas

Romano, Giovanna

Romerosa, Antonio

Rottinger, Eric

Rourke, Wade

Rousseau, Marthe

Roussis, Vassilios

Rovero, Paolo

Russo, Gian Luigi

Rustad, Turid

Saito, Naoki

Sakamoto, Toshio

Salierno, James D.

Salomon, Christine

Samaras, Petros

Sammut, Ivan A.

Sánchez-Moreno, Manuel

Sandjo, Louis Pergaud

Santiago-Vazquez, Lory

Saravanan, Palaniappan

Sarmento, Hugo

Sass, Henrik

Sasso, Severin

Satin, Jonathan

Satomi, Yoshiko

Savvidis, Yiannis

Sayanova, Olga

Scharf, Daniel H.
Schenkel, Johannes

Schmid, Andreas

Schmidt, Boris

Schmidt, Mariane

Schmitz, Ruth

Schneider, Claus

Schock, Tracey

Scholz, Bettina

Schröder, Heinz C.

Schuhmann, Holger

Schuller, Kathryn A.

Schulz, Joseph R.

Schunck, Wolf-Hagen

Schuster, Daniela

Schwindt, Adam

Seabra, Vítor

Seca, Ana

Ségui, Bruno

Seifert, Karlheinz

Sellner, Kevin

Seo, Jeong-Woo

Sérgio, Albuquerque

Serive, Benoit

Sewald, Norbert

Seymour, Justin R.

Shaaban, Mohamed

Sharma, Sushil

Shea, Tom

Sherman, David

Sheu, Jyh-Horng

Shin-ya, Kazuo

Sieber, Jonas

Siegel, Dionicio

Sieiro, Carmen

Sikorska, Hanna

Silipo, Alba

Silva, Luís

Silva, Tiago H.

Simmet, Thomas

Simpson, Thomas J.

Sims, James

Sinclair, Alex 
Sinclair, Andrew

Singh, Rajesh

Sire, Olivier

Slattery, Marc

Smith, G. Jason

Smith, Renee J.

Smith, Terry K.

Smith-Palmer, Truis

Smyth, Thomas

Sneed, Jennifer

Solecka, Jolanta

Song, Peter I.

Song, Zhihong

Soule, Tanya

Souto, María L.

Spahn, Viola

Spiteller, Peter

Spoering, Amy

Srivastava, S.K.

Staerk, Dan

Stanstrup, Jan

Staudinger, Jeff L.

Stensvag, Klara

Stern, Robert

Steverding, Dietmar

Stierle, Andrea

Stierle, Donald

Stout, Elizabeth

Strathmann, Frederick G.

$\mathrm{Su}$, Jui-Hsin

$\mathrm{Su}$, Xiong

Subramani, Ramesh

Suchana, Apple

Suenaga, Kiyotake

Süssmuth, Roderich

Sugiyama, Tetsuya

Sugiyama, Tsuyoshi

Sugumaran, Manickam

Sumarah, Mark

Sung, Ping-Jyun

Sun-Ju, Kim

Suzuki, Hodaka
Svenson, Johan

Sydnes, Magne O.

Szentmiklosi, Jozsef

Taglialatela-Scafati, Orazio

Takahashi, Koretaro

Takaichi, Shinichi

Takemori, Hiroshi

Taketani, Rodrigo Gouvêa

Tanaka, Jun'ichi

Tanaka, Sachiko

Tanaka, Takuji

Tang, Feng-Yao

Tang, Hong

Tantillo, Dean

Tapper, Brian

Tarantini, Francesca

Tarapore, Rohinton S.

Tarman, Kustiariyah

Tasker, Andrew

Tatituri, Raju Venkata Veera

Teas, Jane

Teichert, Russell W.

Teijido, Oscar

Tejesvi, Mysore V.

Temeyer, Kevin B.

Teruya, Kiichiro

Tezuka, Yasuhiro

Thalhammer, Theresia

Thany, Steeve

Theodorakis, Emmanuel A.

Thessen, Anne

Thiel, Teresa

Thiéry, Valérie

Thomas, Olivier P.

Thomas, Torsten

Thomes, Paul

Tobe, Kerstin

Toida, Toshihiko

Toma, Lucio

Tomás, Juan

Tominaga, Akira

Tomm, Janina M.
Tosi, Solveig

Tosti, Elisabetta

Tricone, Antonio

Trischman, Jackie

Trosko, James E.

Trottein, François

Tsuda, Masashi

Turco, Gianluca

Twiner, Michael J.

Tytgat, Jan

Udenigwe, Chibuike C.

Ueda, Katsuhiro

Uhrin, Dusan

Ulens, Chris

Usami, Yoshihide

Usov, Anatoly I.

Usuki, Yoshinosuke

Utkina, Natalia K.

Valdiglesias, Vanessa

Vale, Paulo

Van Mooy, Benjamin A.S.

Van Pée, Karl-Heinz

Van Wagoner, Ryan M.

Varela, João

Varotti, Fernando De Pilla

Varticovski, L.

Varvounis, George

Vasconcelos, Vitor

Velkov, Tony

Venable, Mark E.

Venier, Paola

Vennekens, Rudi

Véron, Benoît

Vetvicka, Vaclav

Viaggiu, Emanuela

Vian, Maryline Abert

Viana, Gustavo H.R.

Vicente, Jan

Vidoudez, Charles

Vignisse, Julie

Vilariño, Natalia

Vinogradov, Evgeny 
Vitetta, Luis

Von Elert, Eric

Von Schacky, Clemens

Wade, John D.

Wahlsten, Matti

Wakimoto, Toshiyuki

Walsh, Catherine J.

Walsh, David

Wang, Shu

Wang, Tianfang

Wang, Wei-Hsien

Wang, Yan-Hua

Warren, C. R.

Warren, J. David

Watabe, Shugo

Watts, Katharine

Wei, Mei

Weiss, John

Weißflog, Jerrit

Wen, Zhi-Hong

Wertz, Philip

Westermann, Bernhard

Westwell, Andrew D.

Wever, Ron

White, Robert L.

Wickenden, Alan
Wijesekara, Isuru

Williams, David

Williams, Robert M.

Wilson, Peter D

Winberg, Pia

Wittlin, Sergio

Witulski, Bernhard

Wolberg, Alisa S.

Wörmer, Lars

Wright, Christine E.

Wu, Shih-Hsiung

Wu, Yang-Chang

Yamashita, Eiji

Yan, Aixin

Yang, Boo

Yang, Hsin-Ling

Yang, Yu-Liang

Yang, Zhicai

Yarotskyy, Viktor

Ye, Tao

Yih, Ling-Huei

Yoshida, Hiroshi

Yoshikawa, Kazuko

Yotsu-Yamashita, Mari

Yuan, Tao

Zachariae, Ulrich
Zahler, Stefan

Zähringer, Ulrich

Zakarian, Armen

Zaki, Noha M.

Zampella, Angela

Zan, Jindong

Zanchetta, Philippe

Zeeck, Axel

Zengler, Karsten

Zervou, Maria

Zhang, Dahai

Zhang, Hongliang

Zhang, Junzeng

Zhang, Xun

Zhang, Yong

Zheng, Qi-Huang

Zhou, Edward

Zhou, Mei

Zhou, Yu-Dong

Zöller, Margot

Zorzano, Antonio

Zotchev, Sergey

Zubía, Eva

(C) 2014 by the authors; licensee MDPI, Basel, Switzerland. This article is an open access article distributed under the terms and conditions of the Creative Commons Attribution license (http://creativecommons.org/licenses/by/3.0/). 